\title{
Trends in breast reconstruction: Implications for the National Health Insurance Service
}

\author{
Ki Yong Hong ${ }^{1}$, Yoosung Son ${ }^{2}$, Hak Chang ${ }^{2}$, Ung Sik Jin ${ }^{2}$ \\ ${ }^{1}$ Department of Plastic and Reconstructive Surgery, Dongguk University Ilsan Hospital, Goyang; ${ }^{2}$ Department of Plastic and Reconstructive \\ Surgery, Seoul National University Hospital, Seoul, Korea
}

Background Breast reconstruction has become more common as mastectomy has become more frequent. In Korea, the National Health Insurance Service (NHIS) began covering breast reconstruction in April 2015. This study aimed to investigate trends in mastectomy and breast reconstruction over the past 10 years and to evaluate the impact of NHIS coverage on breast reconstruction.

Methods Nationwide data regarding mastectomy and breast reconstruction were collected from the Korean Breast Cancer Society registry database. Multiple variables were analyzed in the records of patients who underwent breast reconstruction from January 2005 to March 2017 at a single institution.

Results At Seoul National University Hospital, the total number of reconstruction cases increased 13-fold from 2005 to 2016. The proportion of immediate breast reconstruction (IBR) cases out of all cases of total mastectomy increased from 4\% in 2005 to $52.0 \%$ in 2016. The proportion of delayed breast reconstruction (DBR) cases out of all cases of breast reconstruction and the overall number of DBR cases increased from 8.8\% (20 cases) in 2012 to 18.3\% (76 cases) in 2016. After NHIS coverage was initiated, the proportions of IBR and DBR showed statistically significant increases $(P<0.05)$. Among the IBR cases, the percentage of prosthesisbased reconstructions increased significantly $(P<0.05)$, but this trend was not found with DBR. Total mastectomy became significantly more common after the expansion of NHIS coverage $(\mathrm{P}<0.05)$.

Conclusions Over the last decade, there has been an increase in mastectomy and breast reconstruction, and the pace of increase accelerated after the expansion of NHIS coverage. It is expected that breast reconstruction will be a routine option for patients with breast cancer under the NHIS.

Keywords Mammaplasty / Breast neoplasms / Insurance
Correspondence: Ung Sik Jin Department of Plastic and

Reconstructive Surgery, Seoul National University Hospital, 101

Daehak-ro, Jongno-gu, Seoul 03080, Korea

Tel: $+82-2-2072-2375$

Fax: +82-2-3675-7792

E-mail: usj1011@snu.ac.kr

Received: 31 Aug 2017 • Revised: 2 Mar 2017 • Accepted: 15 Mar 2017

pISSN: 2234-6163 • elSSN: 2234-6171 • https://doi.org/10.5999/aps.2017.01417• Arch Plast Surg 2018;45:239-245

\section{INTRODUCTION}

Breast cancer, which accounts for $15.4 \%$ of all cancers in women, is the second most common malignancy in women in Korea $[1,2]$. The number of patients with breast cancer in Korea was
20,159 in 2013, which is more than three times higher than the corresponding number 10 years previously $[1,2]$. The number of mastectomies performed is steeply increasing in proportion to the increase in breast cancer diagnoses [1,2]. The mortality rate of breast cancer is relatively low compared with other can- 
cers due to early detection and improvements in treatment modalities [1].

Breast reconstruction after curative mastectomy for breast malignancy has gained popularity owing to the high survival rate and the aesthetic superiority of the reconstructed breast $[3,4]$. Thus, both the overall number of breast reconstructions and its ratio per mastectomy have increased over the last decade. Although the personal and social perceptions of breast reconstruction have favorably changed, the high cost of breast reconstruction has functioned as a barrier to choosing this form of surgery [5].

In the United States, the Women's Health and Cancer Rights Act was enacted in 1998 to provide economic coverage for the medical and surgical costs of mastectomy and breast reconstruction [6]. With this legislation, the rates of breast reconstruction increased 2- to 4-fold, depending on socioeconomic status and the type of insurance [5,6]. In Korea, the National Health Insurance Service (NHIS) began to cover breast reconstruction after total mastectomy in April 2015 [7]. It was expected that this change in health care system coverage would have a considerable impact on breast reconstruction trends.

The present study aimed to investigate trends over the past 10 years in mastectomy and breast reconstruction, and compared several variables associated with breast reconstruction before and after the expansion of insurance coverage in order to predict future trends in breast reconstruction.

\section{METHODS}

\section{Data sources}

For nationwide information associated with breast cancer, "Breast Cancer Facts and Figures" issued by the Korean Breast Cancer Society (KBCS) and the KBCS registry database were used; these sources include information about the annual number of patients with breast cancer and the number of breast cancer operations, including breast-conserving surgery (BCS), total mastectomy, and breast reconstruction, in Korea. Data on the number and type of mastectomies performed at Seoul National University Hospital were gathered from the annual report issued by our breast care center.

For a detailed analysis of breast reconstruction, we performed a retrospective study of 2,032 patients who underwent breast reconstruction from January 2005 to March 2017 at a single institution. The date of the operation, sex, age, pathologic diagnosis, type of mastectomy, and the timing and method of breast reconstruction were included in the analysis. The medical records of all patients were reviewed with the approval of the Institutional Review Board (IRB No. H-1602-132-744).

\section{Treatment protocols}

At our institution, breast cancer operations, including BCS and total mastectomy, were performed by general surgeons. In patients undergoing total mastectomy, breast reconstruction was performed by plastic surgeons. The reconstructive procedures not only included autologous tissue reconstruction using a transverse rectus abdominis myocutaneous (TRAM) flap or a latissimus dorsi (LD) muscle flap, but also prosthesis-based reconstructions, including 1-stage implant insertion or the 2-stage expander-implant method, depending on the amount of breast skin that was excised.

\section{Statistical analysis}

All data were queried with Microsoft Excel (Microsoft Corp., Redmond, WA, USA). Associations between clinical variables and the expansion of NHIS coverage were assessed using the chi-square test and the independent t-test. All statistical analyses were performed with SPSS version 22.0 (IBM Corp., Armonk, NY, USA). Results were considered significant at a P-value of 0.05 .

\section{RESULTS}

The incidence of female breast cancer in Korea has steadily increased from 11,339 in 2005 to 20,159 in 2013 [1]. According to the KBCS registry data, the annual number of breast cancer operations has shown an upward trend, increasing from 5,328 cases in 2004 to 12,522 cases in 2013, an approximately 2.4-fold increase $[1,2,8-12]$. The number of breast reconstructions increased from 536 cases in 2004 to 1,279 cases in 2014, an approximately 2.4-fold increase (Fig. 1) [1].

Similarly to the overall trend in Korea, the annual number of breast cancer operations at our institution increased from 803 cases in 2005 to 1,596 cases in 2016, a 2-fold increase. The number of breast reconstructions also showed an upward trend, increasing from 31 cases in 2005 to 415 cases in 2016, an approximately 13-fold increase. Immediate breast reconstruction (IBR), which constituted only $41.9 \%$ of all breast reconstructions in 2005, rapidly became much more common after 2011, accounting for $92.3 \%$ of all breast reconstructions in 2014 (Fig. 2).

The proportion of IBR, calculated as the number of IBR cases divided by the number of total mastectomies, has annually increased from $4 \%$ in 2005 to $38.3 \%$ in 2014 . The proportions were $39.4 \%$ ( 52 cases) from January to March 2015 and $50.8 \%$ (285 cases) from April to December 2015. In 2016, a total of 339 cases were performed, representing an overall reconstruction rate of $52.0 \%$ (Fig. 3). The proportion of delayed breast reconstruction (DBR) cases, calculated as the number of DBR 


\section{Fig. 1. Trends of breast reconstruction in Korea}

Data on the number of breast reconstructions in Korea were collected from the annual Korean Breast Cancer Society registry.

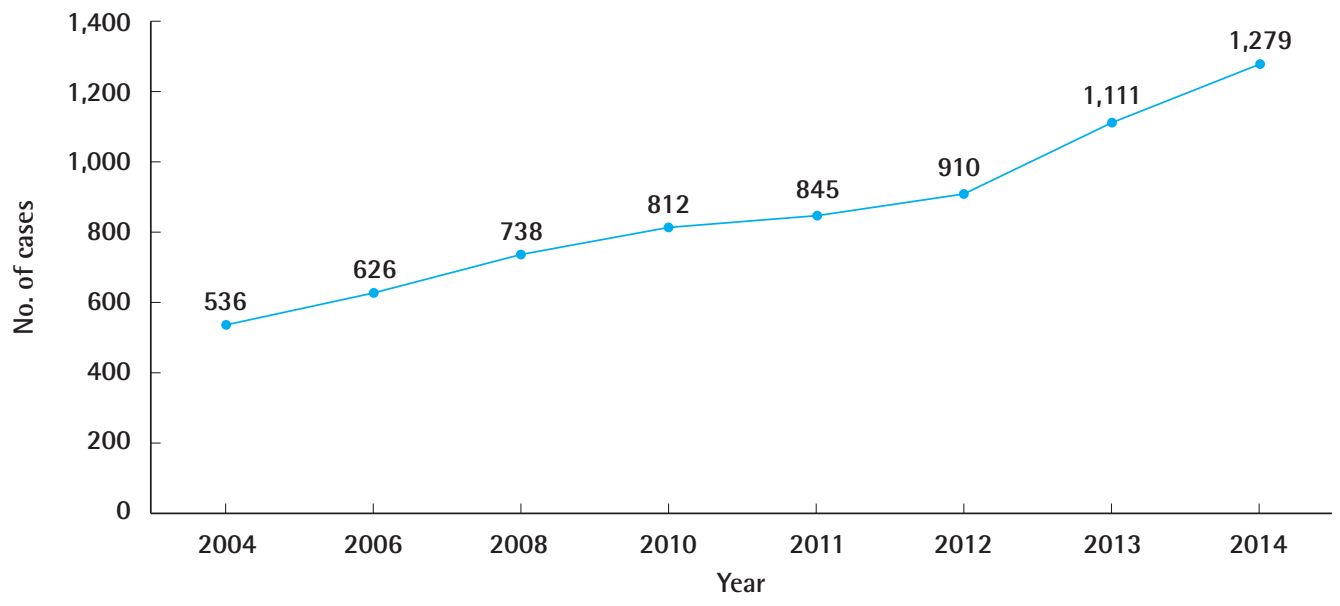

\section{Fig. 2. Trends in type of breast reconstruction}

Cases of breast reconstruction, including immediate and delayed reconstruction, at Seoul National University Hospital were retrospectively reviewed. The number of breast reconstructions has rapidly increased since 2011.

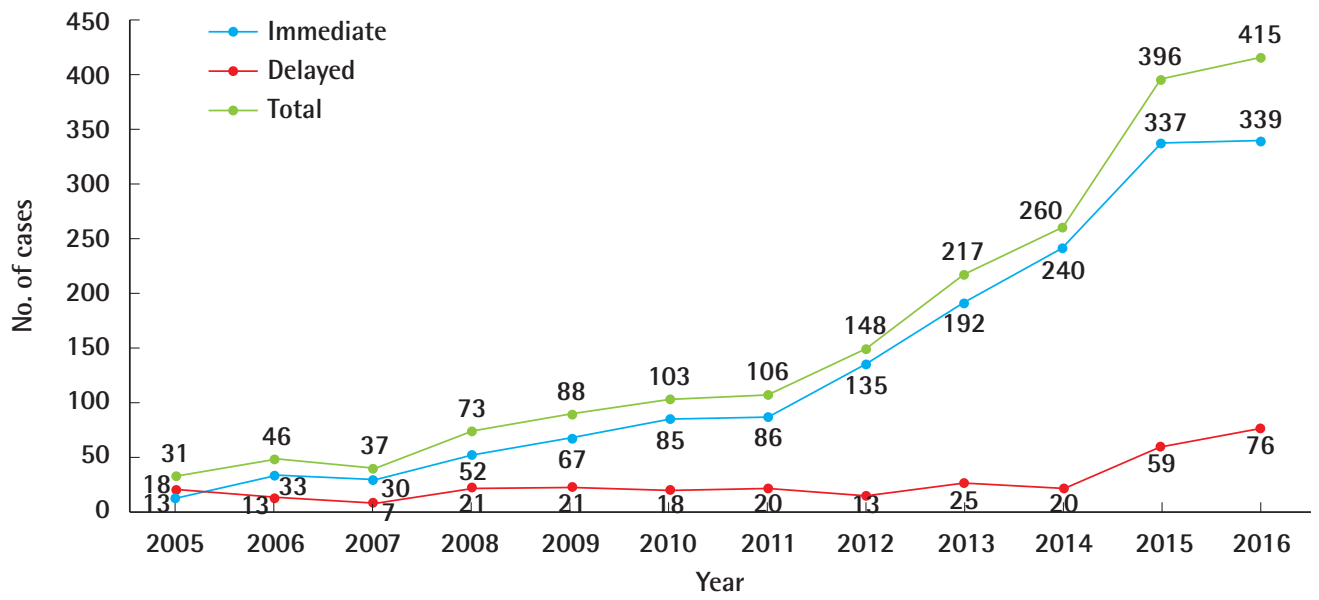

cases divided by the number of total breast reconstructions, was $58.1 \%$ ( 18 cases) in 2005. The number of DBR cases remained relatively constant until 2014 (Fig. 2). As the number of IBR cases increased, its proportion decreased to $7.7 \%$ in 2014, with only 20 total cases. However, after NHIS coverage began in April 2015, the total number of DBR cases rapidly increased to 53 between April and December 2015 and 76 in 2016, representing $15.7 \%$ and $18.3 \%$ of all cases, respectively (Fig. 4).

Within the cases of IBR, the proportions of autologous tissue and prosthesis-based reconstructions were compared. The proportion of autologous tissue reconstructions showed a decreasing trend, accounting for $50.8 \%$ of cases in $2014,35.7 \%$ in 2015 , and $28.0 \%$ in 2016; that of prosthesis-based reconstructions showed an increasing trend, from $49.2 \%$ in 2014 and $64.3 \%$ in 2015 to $72.0 \%$ in 2016 (Fig. 5). The proportion of DBR procedures using autologous tissue reconstruction remained greater than $66.7 \%$, despite showing fluctuations over the years.

To further analyze the effect of the expansion of NHIS coverage, we conducted a comparative analysis of mastectomies and breast reconstructions performed during the 2-year periods before versus after the expansion of insurance coverage (April 2013 to March 2015 vs. April 2015 to March 2017). There was no statistically significant difference in the age distribution of patients between these 2 periods, but the number of patients 


\section{Fig. 3. Rates of IBR after total mastectomy}

Among the procedures performed at our institution, the proportion of immediate breast reconstruction (IBR) cases was measured by dividing the number of IBR cases by the number of total mastectomy cases. The IBR rate increased steeply after the introduction of National Health Insurance Service (NHIS) coverage.

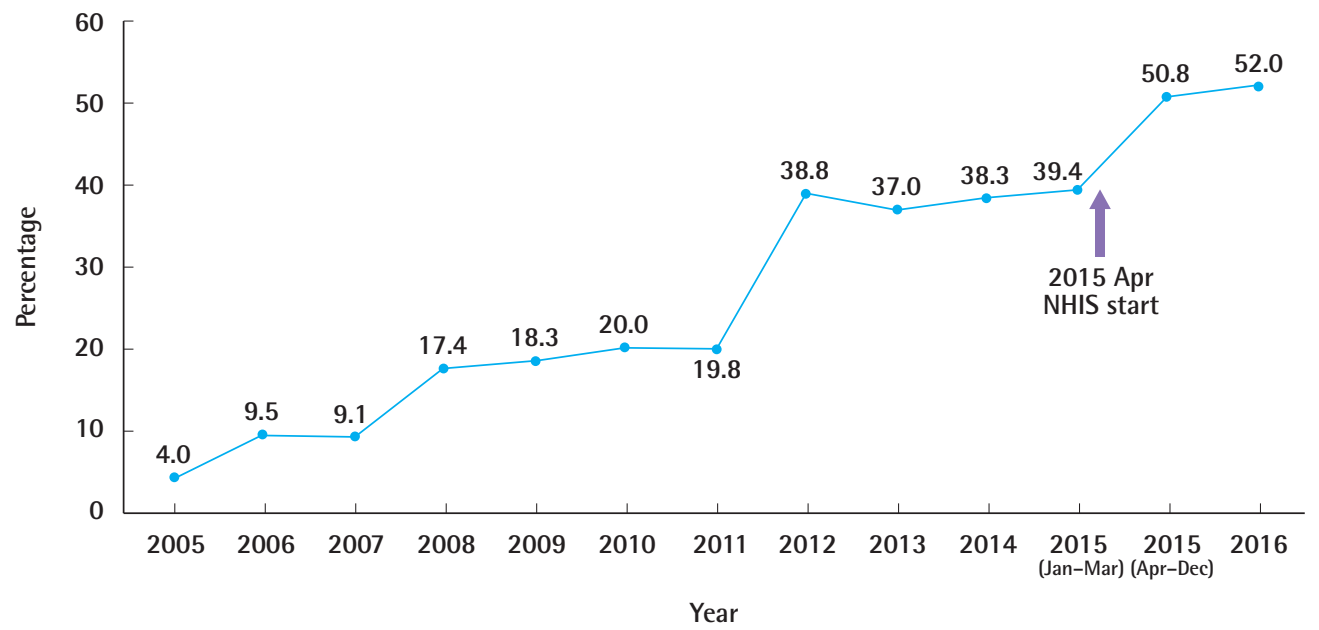

\section{Fig. 4. Proportions of delayed breast reconstructions}

Among the procedures performed at our institution, the proportion of delayed breast reconstruction (DBR) cases was measured by dividing the number of DBR cases by the number of total breast reconstruction cases. As the number of DBR cases increased, the proportion of DBR also increased, reaching $18.3 \%$ after the implementation of coverage by the National Health Insurance Service (NHIS).

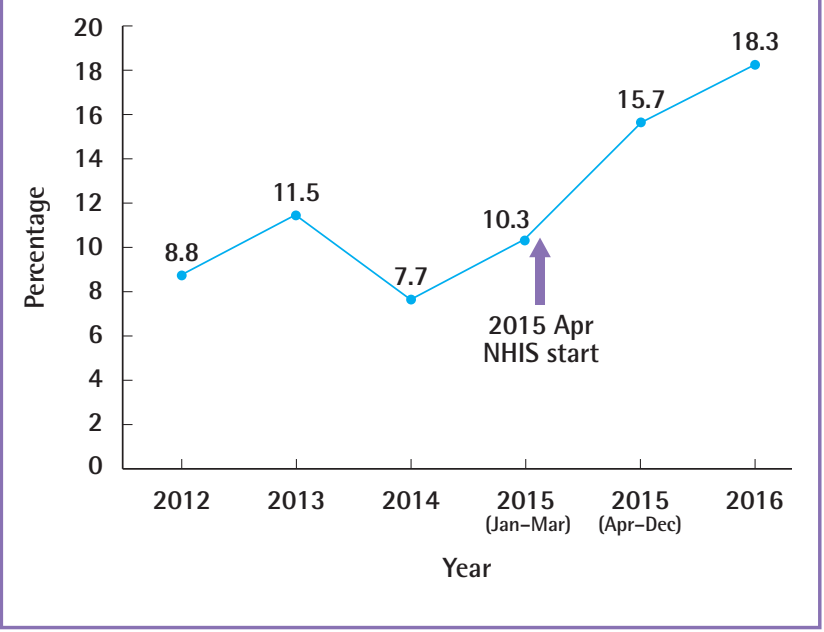

over 50 years old was significantly higher after NHIS expansion $(\mathrm{P}=0.007)$. The proportions of DBR and prosthesis-based reconstruction in IBR after the expansion of insurance coverage were significantly higher than before insurance coverage ( $\mathrm{P}=0.001$ and $\mathrm{P}<0.001$, respectively) (Table 1 ). The proportion of IBR showed a statistically significant increase after the implementation of insurance coverage $(\mathrm{P}<0.001)$ (Table 2).
Table 1. Univariate analysis of clinical variables in patients undergoing breast reconstruction before and after the expansion of NHIS coverage

\begin{tabular}{|c|c|c|c|}
\hline Variable & $\begin{array}{l}\text { Before NHIS } \\
\quad(n=503)\end{array}$ & $\begin{array}{l}\text { After NHIS } \\
(n=908)\end{array}$ & P-value \\
\hline \multicolumn{3}{|l|}{ Age (yr) } & $0.452^{a)}$ \\
\hline Mean \pm SD & $44.9 \pm 8.4$ & $46.8 \pm 8.1$ & \\
\hline Range & $16-70$ & $21-74$ & \\
\hline \multicolumn{3}{|l|}{ Age distribution } & $0.007^{b)}$ \\
\hline$<50$ & $357(71.0)$ & $580(63.9)$ & \\
\hline$\geq 50$ & $146(29.0)$ & $328(36.1)$ & \\
\hline \multicolumn{3}{|l|}{ Reconstruction timing } & $0.001^{\text {b) }}$ \\
\hline Immediate & $451(89.7)$ & 758 (83.5) & \\
\hline Delayed & $52(10.3)$ & $150(16.5)$ & \\
\hline \multicolumn{4}{|l|}{ Reconstruction type } \\
\hline \multicolumn{3}{|l|}{ Immediate } & $<0.001^{\text {b) }}$ \\
\hline Autologous tissue & $250(55.4)$ & $246(32.5)$ & \\
\hline Prosthesis & $201(44.6)$ & $512(67.5)$ & \\
\hline \multicolumn{3}{|l|}{ Delayed } & $0.714^{\text {b) }}$ \\
\hline Autologous tissue & 40 (76.9) & $119(79.3)$ & \\
\hline Prosthesis & $12(23.1)$ & $31(20.7)$ & \\
\hline \multicolumn{4}{|c|}{$\begin{array}{l}\text { Values are presented as number (\%). } \\
\text { NHIS, National Health Insurance Service; SD, standard deviation. } \\
\text { alIndependent t-test; }{ }^{b} \text { Chi-square test. }\end{array}$} \\
\hline
\end{tabular}

After the expansion of NHIS coverage, total mastectomy became significantly more common $(\mathrm{P}<0.001)$ (Table 3$)$.

\section{DISCUSSION}

Recently, as the incidence of breast cancer has increased both in Korea and worldwide, the number of curative operations and 


\section{Fig. 5. Trends of reconstruction methods in IBR}

In the immediate breast reconstruction (IBR) procedures performed in our institution, the proportions of autologous tissue methods and prosthesis-based methods were compared. Autologous tissue reconstruction showed a decreasing trend, whereas prosthesis-based reconstruction showed an increasing trend.

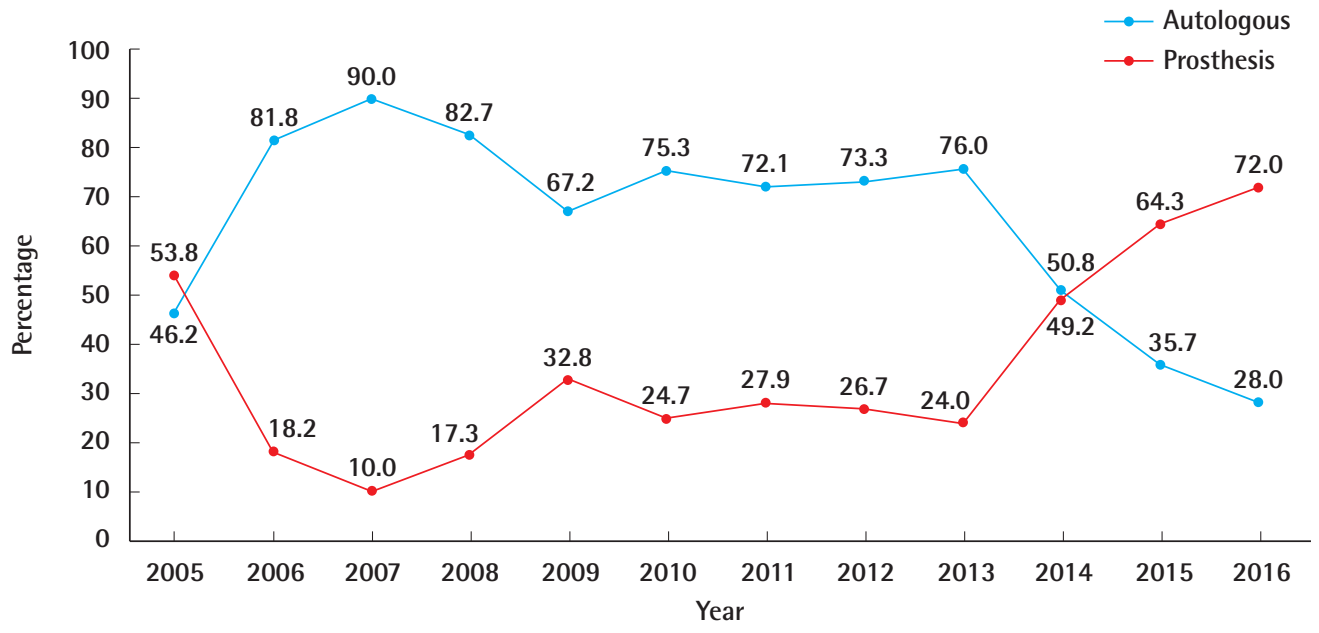

Table 2. Univariate analysis of IBR after total mastectomy before and after the expansion of NHIS coverage

\begin{tabular}{|c|c|c|c|}
\hline Variable & $\begin{array}{c}\text { Before NHIS } \\
(n=1,194)\end{array}$ & $\begin{array}{l}\text { After NHIS } \\
(n=1,385)\end{array}$ & P-value \\
\hline Immediate reconstruction & & & $<0.001^{\text {a) }}$ \\
\hline No & $743(62.2)$ & $627(45.3)$ & \\
\hline Yes & $451(37.8)$ & $758(54.7)$ & \\
\hline
\end{tabular}

breast reconstructions has increased $[1,13]$. Within the category of curative surgery, the proportion of BCS decreased over the past several years after years of continual increases, while that of total mastectomies increased over recent years after years of decline [1]. This trend for total mastectomy to become more common may be attributed to the complications of radiation therapy, which is usually a necessity with BCS, such as skin discoloration, delayed wound healing, and contracture, as well as the increased prevalence of testing for $B R C A 1$ and $B R C A 2$ oncogenes and the need for prophylactic total mastectomy of the contralateral breast in BRCA-positive patients [14]. This change of surgical patterns has become more obvious since the extension of insurance coverage. Previously, BCS was performed to reduce postoperative deformities of the breast. However, with the popularization of breast reconstruction after the expansion of NHIS coverage and the development of reconstruction techniques that satisfactorily overcome postoperative defects, both breast surgeons and patients have likely become more willing to
Table 3. Univariate analysis of mastectomy before and after the expansion of NHIS coverage

\begin{tabular}{|c|c|c|c|}
\hline Variable & $\begin{array}{c}\text { Before NHIS } \\
(n=3,258)\end{array}$ & $\begin{array}{l}\text { After NHIS } \\
(n=3,303)\end{array}$ & P-value \\
\hline Mastectomy & & & $<0.001^{a)}$ \\
\hline BCS & 2,064 (63.4) & $1,918(58.1)$ & \\
\hline TM & $1,194(36.6)$ & $1,385(41.9)$ & \\
\hline \multicolumn{4}{|c|}{$\begin{array}{l}\text { Values are presented as number (\%). } \\
\text { NHIS, National Health Insurance Service; BCS, breast-conserving surgery; TM, } \\
\text { total mastectomy. } \\
\text { a)Chi-square test. }\end{array}$} \\
\hline
\end{tabular}

choose total mastectomy without the burden of oncologic risk and remnant deformity associated with BCS.

The current trend for an increasing number of breast reconstructions to be performed by plastic surgeons after total mastectomy may be attributed to not only the sheer increase in the total number of cases, but also the fact that more patients have started to seek out breast reconstruction as a result of the increased awareness of breast reconstruction through social media. The increase in patients needing breast reconstruction may be due to the fact that the survival rate after diagnosis has increased significantly over the years, and that breast cancer occurs most frequently in patients in their 40s and 50s, who are socially active $[3,4]$. The significant increase in the number of patients over 50 years old supports this explanation. Another factor to consider is the increasing likelihood of general surgeons to refer cases of breast reconstruction to plastic surgeons. Additionally, the significant deterrent factor of high cost has been reduced by the implementation of breast reconstruction 
coverage by the NHIS; this may have played an important role in patients' increasing desire for breast reconstruction. As discussed above, in the United States, after the passage of the Women's Health and Cancer Rights Act in 1998, health benefits cover all stages of breast reconstruction after mastectomy, surgery, and reconstruction of the contralateral breast for symmetry, and any complications related to mastectomy at all stages. However, the applicability of these benefits may differ depending on the patient's insurance type.

In Korea, breast reconstruction began to be covered for patients undergoing total mastectomy in April 2015 [7]. Breast reconstruction using autologous tissue, prosthesis-based breast reconstruction, nipple-areolar complex (NAC) reconstruction, and additional operations needed due to complications or deformities after reconstruction began to be covered by the NHIS. However, breast reconstruction after prophylactic total mastectomy, surgery for breast symmetry, and tattooing procedures for reconstructed NACs were excluded from NHIS coverage. It will be necessary to discuss whether the NHIS should cover contralateral prophylactic mastectomy or surgery for breast symmetry. As a result of the expansion of NHIS coverage, the costs for surgery, anesthesia, inpatient care, and medication declined by half, and the price of tissue expanders and breast implants, which are required for prosthesis-based reconstruction, was also reduced by half. When comparing the cost between different types of reconstruction methods, the expense per operation is higher for autologous tissue reconstruction than for prosthesis-based reconstruction due to the higher cost of surgery, the longer operating time, and the longer hospitalization period. However, considering that prosthesis-based reconstruction is mainly 2-staged and requires an additional cost for the prosthesis, the total cost of breast reconstruction does not differ greatly between autologous tissue and prosthesis-based reconstruction. Some patients planning on undergoing IBR even scheduled their cancer operations after April 2015 in order to reduce their costs. Among breast cancer survivors who underwent breast cancer surgery in the past and were living with a breast deformity, more patients underwent DBR after April 2015, which may have been due to the reduction in cost. Since DBR is an elective operation, the patient's desire for surgery is important. It can be considered that the number of DBR procedures increased due to the reduced economic burden after the expansion of insurance coverage. This may be a typical effect of insurance coverage.

In the past, most IBR procedures used autologous tissue, but more recently, the proportion of reconstructions using prosthesis has increased. This is in accordance with international trends, and may be explained by improvements in implants, the relatively shorter operating and recovery times, and patients' reluc- tance to undergo operations at other sites in addition to their affected breasts, such as the abdomen for TRAM flaps and the back for LD flaps [15-20]. In addition, as the total number of cases of breast reconstruction rapidly increased due to the effects of insurance coverage, prosthesis-based reconstructions would be expected to have accounted for that increase because they require less time and involve simple techniques. However, in cases of DBR, reconstruction using autologous tissue still accounts for the majority of cases. This may be because in the past, the extent of excised soft tissue was wider and postoperative radiation therapy was more common; contracture caused by scar tissue hinders the role of soft tissue expansion, making reconstruction using a prosthesis more difficult.

This study has several limitations. First, the analysis was based on data from a single institution. Thus, our results may not be fully representative; more studies based on multicenter, nationwide, or international statistics are needed. Second, a comparative analysis based on factors including cancer stage, the number of positive lymph nodes, perioperative adjuvant therapy, and the status of estrogen receptor/progesterone receptors was not included in this study. Finally, surgeon-related factors, such as the surgeon's preferred reconstruction method, and facility-related factors have a great influence on the frequency of breast reconstruction procedures. However, this study did not fully account for these effects.

In conclusion, the high survival rate of patients with breast cancer and the aesthetic superiority of the reconstructed breast contributed to the increased proportion of breast reconstructions after curative mastectomy for breast malignancy, and the cost benefits of breast reconstruction with NHIS coverage are likely to have increased the rate of breast reconstruction per mastectomy. In light of this trend, plastic surgeons should make efforts to reduce complications related to reconstructions, to improve breast symmetry, and to strengthen their partnership with general surgeons. Additionally, patients should be educated about their insurance coverage and the benefits of breast reconstruction. In the future, breast reconstruction will be an easily accessible treatment option for patients with breast cancer under the NHIS.

\section{NOTES}

\section{Conflict of interest}

No potential conflict of interest relevant to this article was reported.

\section{Ethical approval}

This study was approved by the Institutional Review Board of 
Seoul National University Hospital (IRB No. H-1602-132-744) and performed in accordance with the principles of the Declaration of Helsinki. Written informed consents were obtained.

\section{REFERENCES}

1. Korean Breast Cancer Society. 2016 Breast cancer facts \& figures. Seoul: Korean Breast Cancer Society; 2016.

2. Min SY, Kim Z, Hur MH, et al. The basic facts of Korean breast cancer in 2013: results of a nationwide survey and breast cancer registry database. J Breast Cancer 2016;19:1-7.

3. Hu ES, Pusic AL, Waljee JF, et al. Patient-reported aesthetic satisfaction with breast reconstruction during the long-term survivorship period. Plast Reconstr Surg 2009;124:1-8.

4. Yueh JH, Slavin SA, Adesiyun T, et al. Patient satisfaction in postmastectomy breast reconstruction: a comparative evaluation of DIEP, TRAM, latissimus flap, and implant techniques. Plast Reconstr Surg 2010;125:1585-95.

5. Yang RL, Newman AS, Lin IC, et al. Trends in immediate breast reconstruction across insurance groups after enactment of breast cancer legislation. Cancer 2013;119:2462-8.

6. American Cancer Society. Women's Health and Cancer Rights Act [Internet]. Atlanta, GA: American Cancer Society [cited 2018 Mar 27]. Available from: https://www.cancer. org/treatment/finding-and-paying-for-treatment/understandinghealth-insurance/health-insurance-laws/womens-healthand-cancer-rights-act.html.

7. Ministry of Health and Welfare. Details on the criteria and method of application of allowance of medical care_revision [Internet]. Sejong: Ministry of Health and Welfare [cited 2018 Mar 27]. Available from: http://www.mohw.go.kr/ react/index.jsp.

8. Ko BS, Noh WC, Kang SS, et al. Changing patterns in the clinical characteristics of Korean breast cancer from 19962010 using an online nationwide breast cancer database. J Breast Cancer 2012;15:393-400.

9. Jung J. Nationwide Korean breast data of 2004 using breast cancer registration program.J Breast Cancer 2006;9:151-61. 10. Jung YS, Na KY, Kim KS, et al. Nation-wide Korean breast cancer data from 2008 using the breast cancer registration program. J Breast Cancer 2011;14:229-36.

11. Kim Z, Min SY, Yoon CS, et al. The basic facts of Korean breast cancer in 2011: results of a nationwide survey and breast cancer registry database. J Breast Cancer 2014;17:99106.

12. Kim Z, Min SY, Yoon CS, et al. The basic facts of Korean breast cancer in 2012: results from a nationwide survey and breast cancer registry database. J Breast Cancer 2015;18:10311.

13. American Society of Plastic Surgeons. 2016 Plastic surgery statistics [Internet]. Arlington Heights, IL: The Society [cited 2018 Mar 27]. Available from: https://www.plasticsurgery. org/news/plastic-surgery-statistics?sub=2016+Plastic+Surge ry+Statistics.

14. Adesiyun TA, Lee BT, Yueh JH, et al. Impact of sequencing of postmastectomy radiotherapy and breast reconstruction on timing and rate of complications and patient satisfaction. Int J Radiat Oncol Biol Phys 2011;80:392-7.

15. Farhangkhoee H, Matros E, Disa J. Trends and concepts in post-mastectomy breast reconstruction. J Surg Oncol 2016; 113:891-4.

16. Albornoz CR, Bach PB, Mehrara BJ, et al. A paradigm shift in U.S. breast reconstruction: increasing implant rates. Plast Reconstr Surg 2013;131:15-23.

17. Cemal Y, Albornoz CR, Disa JJ, et al. A paradigm shift in U.S. breast reconstruction: part 2. the influence of changing mastectomy patterns on reconstructive rate and method. Plast Reconstr Surg 2013;131:320e-326e.

18. Jagsi R, Jiang J, Momoh AO, et al. Trends and variation in use of breast reconstruction in patients with breast cancer undergoing mastectomy in the United States. J Clin Oncol 2014;32:919-26.

19. Leff DR, Bottle A, Mayer E, et al. Trends in immediate postmastectomy breast reconstruction in the United Kingdom. Plast Reconstr Surg Glob Open 2015;3:e507.

20. Lang JE, Summers DE, Cui H, et al. Trends in post-mastectomy reconstruction: a SEER database analysis. J Surg Oncol 2013;108:163-8. 